\title{
Autoantibodies May Predict Immune- Related Toxicity: Results from a Phase I Study of Intralesional Bacillus Calmette-Guérin followed by Ipilimumab in Patients with Advanced Metastatic Melanoma
}

OPEN ACCESS

Edited by:

José Mordoh,

Leloir Institute Foundation (FIL),

Argentina

Reviewed by:

Derre Laurent,

Centre Hospitalier Universitaire

Vaudois (CHUV), Switzerland

Carlos Alfaro,

Universidad de Navarra, Spain

${ }^{*}$ Correspondence:

Andreas Behren

andreas.behren@onjcri.org.au;

Jonathan Cebon

jonathan.cebon@onjcri.org.au

+Shared senior authorship.

Specialty section:

This article was submitted to Cancer Immunity and Immunotherapy,

a section of the journal

Frontiers in Immunology

Received: 15 December 2017

Accepted: 14 February 2018

Published: 02 March 2018

Citation:

Da Gama Duarte J, Parakh S, Andrews MC, Woods K, Pasam A,

Tutuka C, Ostrouska S,

Blackburn JM, Behren A and

Cebon J (2018) Autoantibodies May

Predict Immune-Related Toxicity:

Results from a Phase I Study of

Intralesional Bacillus Calmette-Guérin

followed by Ipilimumab in Patients with Advanced Metastatic Melanoma.

Front. Immunol. 9:411.

doi: 10.3389/fimmu.2018.00411
Jessica Da Gama Duarte ${ }^{1,2,3}$, Sagun Parakh ${ }^{1,2,3}$, Miles C. Andrews ${ }^{1,2,3,4}$, Katherine Woods ${ }^{1,2,3}$, Anupama Pasam ${ }^{1,2,3}$, Candani Tutuka ${ }^{1,2,3}$, Simone Ostrouska ${ }^{1,2}$, Jonathan M. Blackburn ${ }^{5,6}$, Andreas Behren ${ }^{1,2,3 *+}$ and Jonathan Cebon ${ }^{1,2,3 *+}$

\begin{abstract}
'Olivia Newton-John Cancer Research Institute, Heidelberg, VIC, Australia, ${ }^{2}$ School of Cancer Medicine, La Trobe University, Heidelberg, VIC, Australia, ${ }^{3}$ Ludwig Institute for Cancer Research, Melbourne-Austin Branch, Melbourne, VIC, Australia, ${ }^{4} \mathrm{MD}$ Anderson Cancer Center, University of Texas, Houston, TX, United States, ${ }^{5}$ Department of Integrative Biomedical Sciences and Institute of Infectious Disease and Molecular Medicine, University of Cape Town, Cape Town, South Africa, ${ }^{6}$ Sengenics Corporation, Singapore, Singapore
\end{abstract}

Immune checkpoint inhibitors (ICls) have revolutionized the treatment of advanced melanoma. The first $\mathrm{ICl}$ to demonstrate clinical benefit, ipilimumab, targets cytotoxic T-lymphocyte-associated antigen-4 (CTLA-4); however, the long-term overall survival is just $22 \%$. More than 40 years ago intralesional (IL) bacillus Calmette-Guérin (BCG), a living attenuated strain of Mycobacterium bovis, was found to induce tumor regression by stimulating cell-mediated immunity following a localized and self-limiting infection. We evaluated these two immune stimulants in combination with melanoma with the aim of developing a more effective immunotherapy and to assess toxicity. In this phase I study, patients with histologically confirmed stage III/IV metastatic melanoma received IL BCG injection followed by up to four cycles of intravenous ipilimumab (anti-CTLA-4) (ClinicalTrials.gov number NCT01838200). The trial was discontinued following treatment of the first five patients as the two patients receiving the escalation dose of BCG developed high-grade immune-related adverse events (irAEs) typical of ipilimumab monotherapy. These irAEs were characterized in both patients by profound increases in the repertoire of autoantibodies directed against both self- and cancer antigens. Interestingly, the induced autoantibodies were detected at time points that preceded the development of symptomatic toxicity. There was no overlap in the antigen specificity between patients and no evidence of clinical responses. Efforts to increase response rates through the use of novel immunotherapeutic combinations may be associated with higher rates of irAEs, thus the imperative to identify biomarkers of toxicity remains strong. While the small patient numbers in this trial do not allow for any conclusive evidence of predictive biomarkers, the observed changes warrant further examination of autoantibody repertoires in larger patient cohorts at risk of developing irAEs during their course of treatment. In summary, dose escalation of IL BCG 
followed by ipilimumab therapy was not well tolerated in advanced melanoma patients and showed no evidence of clinical benefit. Measuring autoantibody responses may provide early means for identifying patients at risk from developing severe irAEs during cancer immunotherapy.

Keywords: melanoma, bacillus Calmette-Guerin, ipilimumab, immune-related adverse events, protein microarrays

\section{INTRODUCTION}

The treatment of metastatic melanoma is rapidly evolving with the approval of a number of targeted therapies and immune checkpoint inhibitors (ICIs) in a short period of time. Despite significant improvement in outcomes, the median overall survival of patients with advanced disease remains poor (1). Ipilimumab, a fully humanized IgG1 monoclonal antibody, is the first ICI to show a survival benefit in advanced melanoma in treatmentnaïve and pretreated patients (2). Ipilimumab competitively binds to cytotoxic T-lymphocyte-associated antigen-4 (CTLA-4) more efficiently than B7 molecules found on antigen-presenting cells, preserving CD28 signaling to potentiate antitumor T-cell responses. Side effects from ipilimumab, called immune-related adverse events (irAEs), are typically inflammatory in nature and may relate to the activation of the immune system against self-antigens (3). High-grade 3 or 4 irAEs occur in $10-15 \%$ of patients: primarily colitis, diarrhea, rash, hepatotoxicity, and endocrinopathies $(4,5)$.

Bacillus Calmette-Guérin (BCG) is a living attenuated strain of Mycobacterium bovis that stimulates cell-mediated immunity by producing a localized and self-limiting infection. It has been shown to have antitumor activity in several clinical studies (6-9). The exact mechanism of action is not well known, but it is probable that BCG invokes a local inflammatory response involving a variety of both innate and adaptive immune effector cells (10). Intravesical immunotherapy with BCG has been established as the most effective adjuvant treatment for preventing local recurrences and tumor progression following transurethral resection of nonmuscle invasive bladder cancer. A large number of clinical trials have established a major role for BCG immunotherapy in urological oncology (11-13).

Intralesional (IL) BCG can be effective in inducing the regression of cutaneous metastatic melanoma $(7,9,10,14,15)$. Inflammation and ulceration occurred in most cases, and subsequent regression of the injected lesion was commonly observed. In fewer than $10 \%$ of patients receiving IL BCG, regression of noninjected lesions was seen, and occasional long-term diseasefree survival has been reported $(15,16)$, likely due to persistent T-cell immunity. Side effects were dose-dependent and included largely constitutional flu-like symptoms such as fever and myalgia, generally lasting 8-9 weeks that could be stopped with isoniazid (17).

The safety and efficacy of BCG given in combination with melanoma vaccines were evaluated in several phase I, II and III clinical trials $(9,18-20)$. The multicenter phase III randomized studies of BCG plus a polyvalent melanoma vaccine (CancerVax) versus BCG plus a placebo as a postsurgical treatment for stage
III or IV melanoma (MMAIT-III and MMAIT-IV trials) were stopped when interim analyses demonstrated that it was unlikely that the vaccine would provide significant evidence of a survival benefit. Nevertheless, excellent survival was seen for the entire study population with $42 \%$ of stage IV and $63 \%$ of stage III patients projected to be alive at 5 years (21). This high survival may have been due to selection bias or BCG, which may have acted as an active immunotherapeutic agent at the administered dose.

Despite the success of ICI, a significant proportion of patients either does not respond to treatment or becomes resistant after initial response. This failure of therapy may result from a variety of mechanisms, such as immune ignorance, a hostile tumor environment, alternative immune checkpoint-independent regulatory mechanisms, inadequate antigenicity, or antigen downregulation (22). Strategies that induce a favorable inflammatory tumor microenvironment prior to, or at the time of, ICI have the potential to increase the effectiveness of anticancer immune therapies.

In this study, we evaluated the safety, clinical efficacy, and immunogenicity of IL BCG followed by ipilimumab (supported by the Ludwig Institute for Cancer Research and by Bristol-Myers Squibb; ClinicalTrials.gov number NCT01838200/LUD2012003). Given the possibility of ipilimumab to potentiate the inflammatory effects of BCG, particular attention was paid to the evaluation of local and systemic inflammatory toxicities.

Protein microarrays have been widely used to detect and quantify the presence of autoantibodies in a variety of autoimmune diseases (23). Since patients treated with immunotherapy develop irAEs that resemble autoimmune disease, we further investigated the autoantibody repertoire of all recruited patients to characterize their serological responses as part of our broader immune-monitoring approach.

\section{MATERIALS AND METHODS}

\section{Patients}

Patients of good performance status (Eastern Cooperative Oncology Group score $0-1$ ) with histologically confirmed unresectable stage III or stage IV melanoma were enrolled in the study. The major inclusion criteria included the presence of at least one cutaneous or subcutaneous metastatic lesion amenable to IL therapy. Key exclusion criteria included symptomatic or active cerebral metastases requiring corticosteroids, prior history of tuberculosis, hypersensitivity to BCG or contraindication to the use of isoniazid, autoimmune disease, immunodeficiency disease, or the use of immunosuppressive therapy. 


\section{Study Design}

This was a single-site, open-label phase I, dose-escalation study. Because of the potential for ipilimumab to amplify inflammatory responses mediated by BCG, a variety of safety precautions were taken; eligible patients had a skin test for tuberculin reactivity with purified protein derivative and were enrolled in one of two cohorts, depending on the size of the induration. Patients with an induration of $<10 \mathrm{~mm}$ in diameter were enrolled in cohort 1 which utilized a $3+3$ dose-escalation design. Patients with a reaction of $\geq 10 \mathrm{~mm}$ were enrolled in cohort 2 . Enrollment of the first three patients was staggered by 3 weeks; subsequent patients were enrolled without delay. Patients enrolled in cohort 1, group 1, received $200 \mu \mathrm{l} \mathrm{BCG} \mathrm{(day} \mathrm{1,} \mathrm{D1)} \mathrm{containing}$ $0.16-0.64 \times 10^{6} \mathrm{cfu}$, and patients in groups 2 and 3 received $200 \mu \mathrm{l}$ BCG containing $0.80-3.20 \times 10^{6} \mathrm{cfu}$ and $4.00-16.00 \times 10^{6} \mathrm{cfu}$, respectively. All patients enrolled in cohort 2 received $200 \mu \mathrm{l} \mathrm{BCG}$ containing $0.16-0.64 \times 10^{6} \mathrm{cfu}$ BCG. To ensure that no active BCG infection was present at the time of ipilimumab administration, oral isoniazid of $300 \mathrm{mg} /$ day was commenced on D29 and continued for 4 weeks in all patients. Ipilimumab was administered intravenously on D36 at a dose of $3 \mathrm{mg} / \mathrm{kg}$ every 3 weeks for a total of four doses. Patients with responding or a stable disease by RECIST v.1.1 or immune-related response criteria (irRC) were scheduled to receive ipilimumab as maintenance therapy administered at a 12-week interval for a further four doses (Figure 1).

This study was conducted at Austin Health, Heidelberg, Australia, and was approved by the institution's human research ethics committee. Written informed consent was obtained from all patients, and all methods were performed in accordance with the protocol-specified guidelines and regulations.

\section{Dose-Limiting Toxicity (DLT)}

Dose-limiting toxicity (DLT) was defined as $\geq$ grade 3 skin reaction at the injection site or $\geq$ grade 3 toxicity associated with BCG administration. All toxicities were graded as per the Common Terminology Criteria for Adverse Events (CTCAE) version 4.0. The protocol included provisions for BCG dose adjustments in the event of grade 3 or 4 toxicities including local skin reactions. DLT of ipilimumab was defined as any toxicity that required dosing modifications in accordance with the recommendations in the product information, or $\geq$ grade 3 hematologic or nonhematologic toxicity.

\section{Safety Assessments: Screening, Baseline, and Follow-up}

Safety assessments were carried out at patient enrollment, D1, D29, D36, D43, D50, D57, D78, D99, D113, D141, and D204.
Target skin lesions were monitored with clinical photography at D1, D36, D113, D141, and D204. Plasma and peripheral blood mononuclear cells were collected at D1, D36, D43, D57, D78, D113, D141, and D204.

\section{Response Assessment}

Tumors were assessed clinically and by contrast-enhanced (CT) scans (brain, neck, chest, abdomen, and pelvis) at patient enrollment, D36, D113, D141, and D204. Tumor response and progression were determined in accordance with RECIST v1.1 criteria (24) and irRC (25).

\section{Autoantibody Profiling Using the Immunome ${ }^{\mathrm{TM}}$ Protein Arrays}

A total of 15 plasma samples were assayed using the Immunome ${ }^{\mathrm{TM}}$ protein array (Sengenics Corporation, Singapore) as previously described (26). These samples were selected to assess the seromic profile associated with the period of BCG treatment and that of ipilimumab treatment separately. The array contains quadruplicate spots of 1,627 full-length, correctly folded, and fully functional immobilized self- and cancer proteins. These include cancer antigens [mainly cancer-testis antigens (CTAs)], transcription factors, kinases, signaling proteins, and others (Table S1 in Supplementary Material). Given the expected reactivity toward self-antigens, 19 healthy individual samples were independently assayed as controls. Raw data were processed and normalized using a robust pipeline that has been previously described (27) (Supplementary Methods). Statistical analysis (GraphPad Prism and Microsoft Excel) and data-clustering methods [Multiple experiment Viewer $(\mathrm{MeV})$ ] were then applied to the resulting data and visualized using above significance threshold counts, box plots, comparative size-proportional pie charts, dendrogram, and heat maps.

\section{RESULTS}

\section{Baseline Patient Characteristics}

Between April 2014 and June 2015, five patients were enrolled in the study. Cohort 1 included patients $1-3$ in group 1 who received a $0.16-0.64 \times 10^{6} \mathrm{cfu}$ BCG dose and patients 4 and 5 in group 2 who received a $0.80-3.20 \times 10^{6} \mathrm{cfu}$ BCG dose. No patients were enrolled in planned group 3 or cohort 2 . The mean age of patients was 59 years (range $43-71$ years), with three males (60\%). Two patients had a BRAF mutation and one patient a NRAS mutation. Two patients had prior immunotherapy with an NY-ESO-1

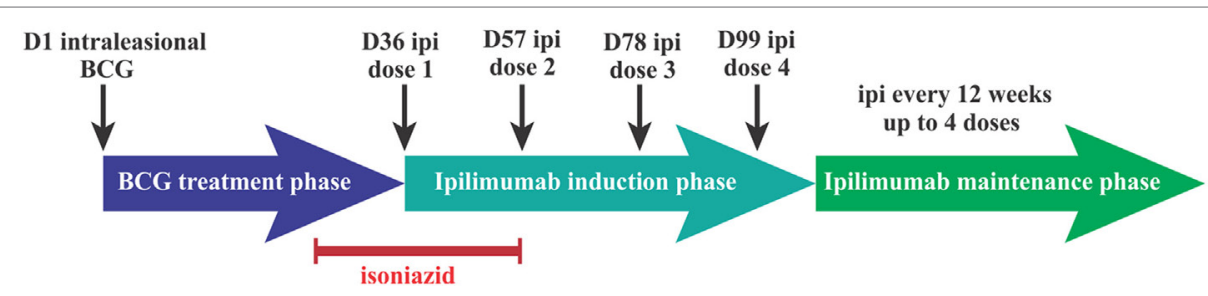

FIGURE 1 | Intralesional bacillus Calmette-Guérin (BCG) followed by ipilimumab phase I trial treatment schedule. Dn, day n; Ipi, ipilimumab. 
vaccine or pembrolizumab, and one patient had undergone prior targeted therapy with a BRAF inhibitor (Table 1).

\section{Safety}

All patients experienced treatment-related adverse events (Table 2). Within cohort 1, group 1 patients displayed grade 1 adverse events, including fatigue, nausea, and pruritus. One patient displayed minor discomfort in the injected lesion site. Both group 2 patients in the BCG dose-escalation cohort also displayed grade 1 events, including pruritus and fatigue, but additionally these patients experienced high-grade irAEs, patient 4 with grade 3 pruritus, rash, and hepatitis on D49, and patient 5 with grade 4 colitis and secondary grade 3 small bowel ileus on D94 (Figure S1 in Supplementary Material). These irAEs were of high grade at the first onset and led to the discontinuation of the study.

\section{Clinical Activity}

Injected lesions progressed in two patients (one resection required, Figure S2 in Supplementary Material), remained stable in two others, and was not evaluated in one patient (taken off study on D49). No clinical responses were observed at noninjected sites of disease; four patients had progressive disease on the basis of RECIST v1.1 criteria and irRC (Figure S3 in Supplementary Material). The remaining patient was taken off study early and did not have tumor measurement assessments. All patients ultimately progressed at sites that included lung, liver, bone, and skin.

\section{Treatment-Induced Changes in the Autoantibody Repertoire}

Plasma samples from five patients were analyzed for autoantibody responses by Immunome $^{\mathrm{TM}}$ protein array at three

TABLE 1 | Baseline patient characteristics.

Patient Gender Stage (AJCC v7)

Prior systemic

First-line treatment

Second-line treatment

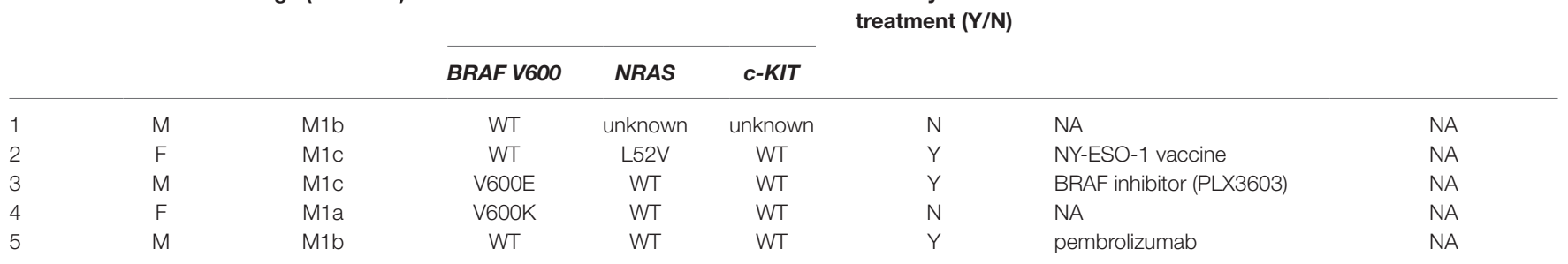

M, male; F, female; WT, wild-type; N, no; Y, yes; NA, not applicable; AJCC v7, American Joint Commission on Cancer classification, version 7.

TABLE 2 | Administered treatment schedule, clinical responses, adverse events, and subsequent treatments across cohort.

\begin{tabular}{|c|c|c|c|c|c|c|c|c|c|c|}
\hline \multirow[t]{2}{*}{ Patient } & \multirow[t]{2}{*}{ Cohort 1} & \multirow{2}{*}{$\begin{array}{l}\text { Bacillus } \\
\text { Calmette- } \\
\text { Guérin (BCG) } \\
\text { dose }\end{array}$} & \multirow{2}{*}{$\begin{array}{l}\text { Site(s) of BCG } \\
\text { injection }\end{array}$} & \multirow{2}{*}{$\begin{array}{l}\text { Number of } \\
\text { ipilimumab } \\
\text { doses } \\
\text { received }\end{array}$} & \multicolumn{2}{|c|}{ Response } & \multirow{2}{*}{$\begin{array}{l}\text { Sites of } \\
\text { progression }\end{array}$} & \multirow[t]{2}{*}{ irAEs } & \multirow[t]{2}{*}{ Other AEs } & \multirow{2}{*}{$\begin{array}{l}\text { Subsequent } \\
\text { treatment }\end{array}$} \\
\hline & & & & & $\begin{array}{l}\text { Injected } \\
\text { lesions }\end{array}$ & $\begin{array}{l}\text { Noninjected } \\
\text { sites }\end{array}$ & & & & \\
\hline 1 & Group 1 & $\begin{array}{l}0.16- \\
0.64 \times 10^{6} \mathrm{cfu}\end{array}$ & Left axilla ${ }^{a}$ & 4 & $\begin{array}{l}\mathrm{PD}- \\
\text { required } \\
\text { resection }\end{array}$ & PD & $\begin{array}{l}\text { Abdominal wall, } \\
\text { pelvic node }\end{array}$ & G1 pruritus & $\begin{array}{l}\text { G1 fatigue, } \\
\text { G1 left } \\
\text { axillary } \\
\text { discomfort }\end{array}$ & Nivolumab \\
\hline 2 & Group 1 & $\begin{array}{l}0.16- \\
0.64 \times 10^{6} \mathrm{cfu}\end{array}$ & Right axillab & 4 & $\mathrm{SD}$ & PD & Bone, liver & 0 & $\begin{array}{l}\text { G1 fatigue, } \\
\text { G1 nausea }\end{array}$ & Nivolumab \\
\hline 3 & Group 1 & $\begin{array}{l}0.16- \\
0.64 \times 10^{6} \mathrm{cfu}\end{array}$ & Left axillary nodule & 2 & $\mathrm{SD}$ & PD & $\begin{array}{l}\text { Pulmonary, } \\
\text { pleura, bone, } \\
\text { and hepatic }\end{array}$ & 0 & G1 fatigue & $\begin{array}{l}\text { Dabrafenib } \\
\text { and trametinib }\end{array}$ \\
\hline 4 & Group 2 & $\begin{array}{l}0.80- \\
3.20 \times 10^{6} \mathrm{cfu}\end{array}$ & Right upper thigh ${ }^{a}$ & 1 & $\mathrm{NE}$ & NE & NE & $\begin{array}{l}\text { G1 diarrhea, } \\
\text { G3 pruritus, } \\
\text { G3 rash, } \\
\text { G3 hepatitis }\end{array}$ & $\begin{array}{l}\text { G1 nausea, } \\
\text { G2 fatigue }\end{array}$ & $\begin{array}{l}\text { Dabrafenib } \\
\text { and trametinib }\end{array}$ \\
\hline 5 & Group 2 & $\begin{array}{l}0.80- \\
3.20 \times 10^{6} \mathrm{cfu}\end{array}$ & $\begin{array}{l}\text { Right anterior chest } \\
\text { wall, right shoulder }\end{array}$ & 3 & PD & PD & $\begin{array}{l}\text { Cutaneous } \\
\text { lesions }\end{array}$ & $\begin{array}{l}\text { G1 pruritus, } \\
\text { G1 rash, } \\
\text { G2 diarrhea, } \\
\text { G3 small } \\
\text { bowel ileus, } \\
\text { G4 colitis }\end{array}$ & G1 fatigue & DTIC \\
\hline
\end{tabular}

irAEs, immune-related adverse events; AEs, adverse events, PD, progressive disease; SD, stable disease; NE, not evaluated; Gn, grade $n$.

aSubcutaneous.

${ }^{b}$ Lymph node. 
distinct available time points: pre-BCG, post-BCG, and post-ipilimumab. Minor variations in sampling time point between patients are negligible, as changes in the autoantibody repertoire remain detectable for at least 90 days, due to the sensitivity of the assay and the expected 30-day half-life of specific autoantibody titers. In addition, plasma samples from 19 anonymized healthy individual sera were assayed to establish antigen-specific significance thresholds. Visual assessment of all slides revealed high-quality printing and slide handling. Data resulting from seven antigens (CASP10, COMMD3, FANCG, SMARCE1, STAT1, TNFRSF11B, and TYR) were excluded from analysis, because replicates were flagged as "noisy" on all slides. High levels of saturation were repeatedly detected against RBPJ for all patient samples, most likely due to its specific binding to the immunoglobulin kappa-type J segment recombination signal sequence. Nonetheless, this antigen was not excluded from analysis. Positive control CV calculations revealed a variation of $3.4 \%$ (cy3-biotin-BSA spots), not requiring data normalization to be performed. The remaining average net intensity autoantibody data against 1,620 antigens was used for downstream analysis. Significance thresholds were calculated as 2 SD from the mean of the data derived from 19 healthy individuals, on an antigen-by-antigen basis (Table S2 in Supplementary Material).

Positive signals above these thresholds and the day of onset of high-grade irAEs are shown in Figure 2. Although normally expressed only in the human germ line, CTAs are aberrantly overexpressed in cancer (28) and enable the assessment of cancerspecific responses alongside the broad autoantibody responses that may be unrelated to cancer immunity. Autoantibody responses according to clinical time lines are shown in Figure 3. Patients $1(n=71 / 1,620), 2(n=34 / 1,620)$, and $3(n=27 / 1,620)$ developed response to relatively few antigens over the period of study. This averaged $3 \%$ of the array content. By contrast, patients $4(n=853 / 1,620)$ and $5(n=505 / 1,620)$ had a substantially larger amount of de novo and induced counts. These represented an average of $42 \%$ of all antigens on the array. This increase was equally evident for both self- and cancer antigens. It is worth noting that enhanced autoantibody reactivity was only seen in the patients who experienced high-grade irAEs (Figure 3B). Furthermore, this broad repertoire was either preexisting at baseline (patient 4) or induced after BCG administration (patient 5) and preceded the development of symptoms in both instances.

In addition, the resulting data were analyzed using the Spearman rank correlation, as a means of assessing sample clustering. When inspecting the resulting dendrogram (Figure 4), all patient time points clustered together on a patient-by-patient basis, serving as an internal validation of the resulting data. Two distinct sample clusters were apparent, adequately separating patients displaying low- and high-grade irAEs. The baseline data for patient 5 were distinct from the remaining time points and from patient 4 , which again highlighted that the boost in excessive autoantibody reactivity only occurred after BCG administration, rather than a

\begin{tabular}{|c|c|c|c|c|}
\hline & & AN1 & GEN T & PE \\
\hline PATIENT & TIME POINT & TOTAL & SELF & CTAS \\
\hline \multirow{3}{*}{ Patient 1} & D43 (start ipi) & 55 & 52 & 3 \\
\hline & D60 (on ipi) & 62 & 58 & 4 \\
\hline & D114 (on ipi) & 55 & 51 & 4 \\
\hline \multirow{3}{*}{ Patient 2} & D1 (start BCG) & 15 & 15 & 0 \\
\hline & D36 (start ipi) & 34 & 32 & 2 \\
\hline & D113 (on ipi) & 11 & 11 & 0 \\
\hline \multirow{3}{*}{ Patient 3} & D1 (start BCG) & 26 & 26 & 0 \\
\hline & D56 (on ipi) & 5 & 5 & 0 \\
\hline & D75 (on ipi) & 21 & 21 & 0 \\
\hline \multirow{3}{*}{ Patient 4} & D1 (start BCG) & 342 & 314 & 28 \\
\hline & D35 (start ipi) & 812 & 766 & 46 \\
\hline & D60 (on ipi) & 483 & 459 & 24 \\
\hline \multirow{3}{*}{ Patient 5} & D1 (start BCG) & 13 & 13 & 0 \\
\hline & D44 (start ipi) & 504 & 466 & 38 \\
\hline & D79 (on ipi) & 353 & 323 & 30 \\
\hline
\end{tabular}

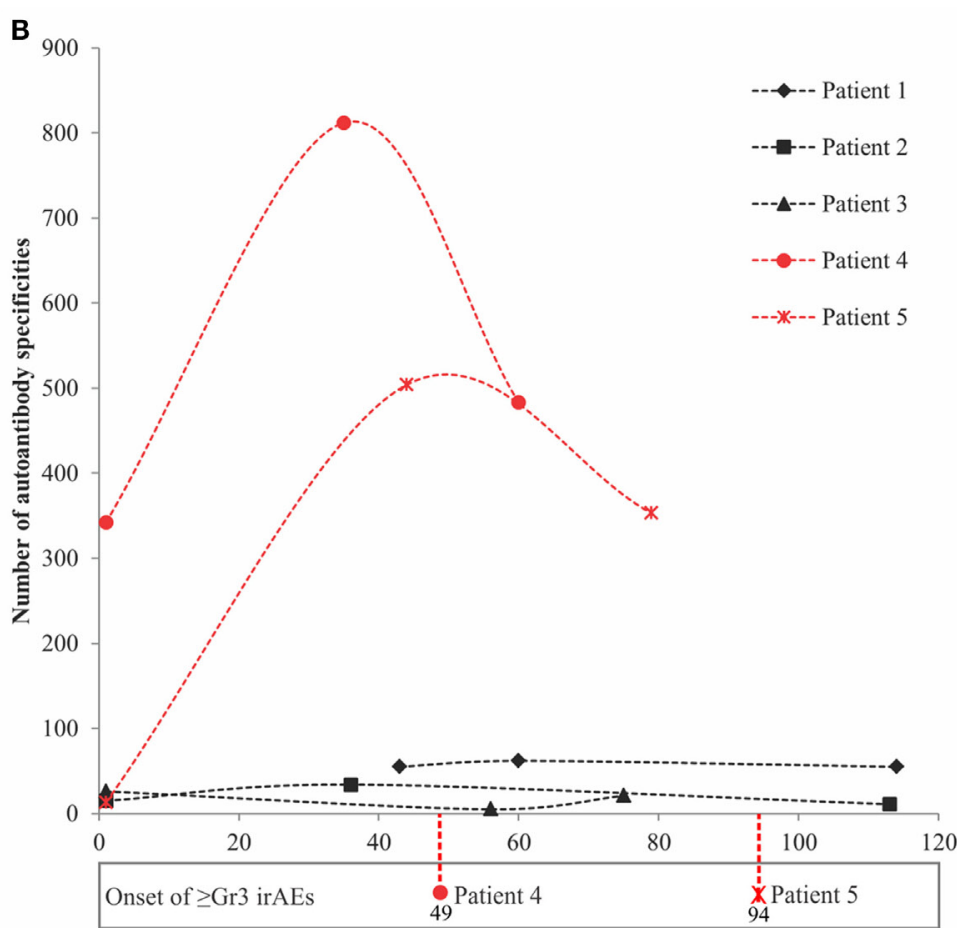

Days after commencement of therapy

FIGURE 2 | Antigen-specific autoantibody counts above healthy individual-derived thresholds of significance. (A) Tabulated counts divided by self-antigens (SELF) and cancer-testis antigens (CTAs). (B) Plotted counts comparing patients who developed high-grade immune-related adverse events (irAEs) (red) versus those who did not (black), along with the day of onset of high-grade irAEs. Dn, day n; Ipi, ipilimumab; Gr3, grade 3. 

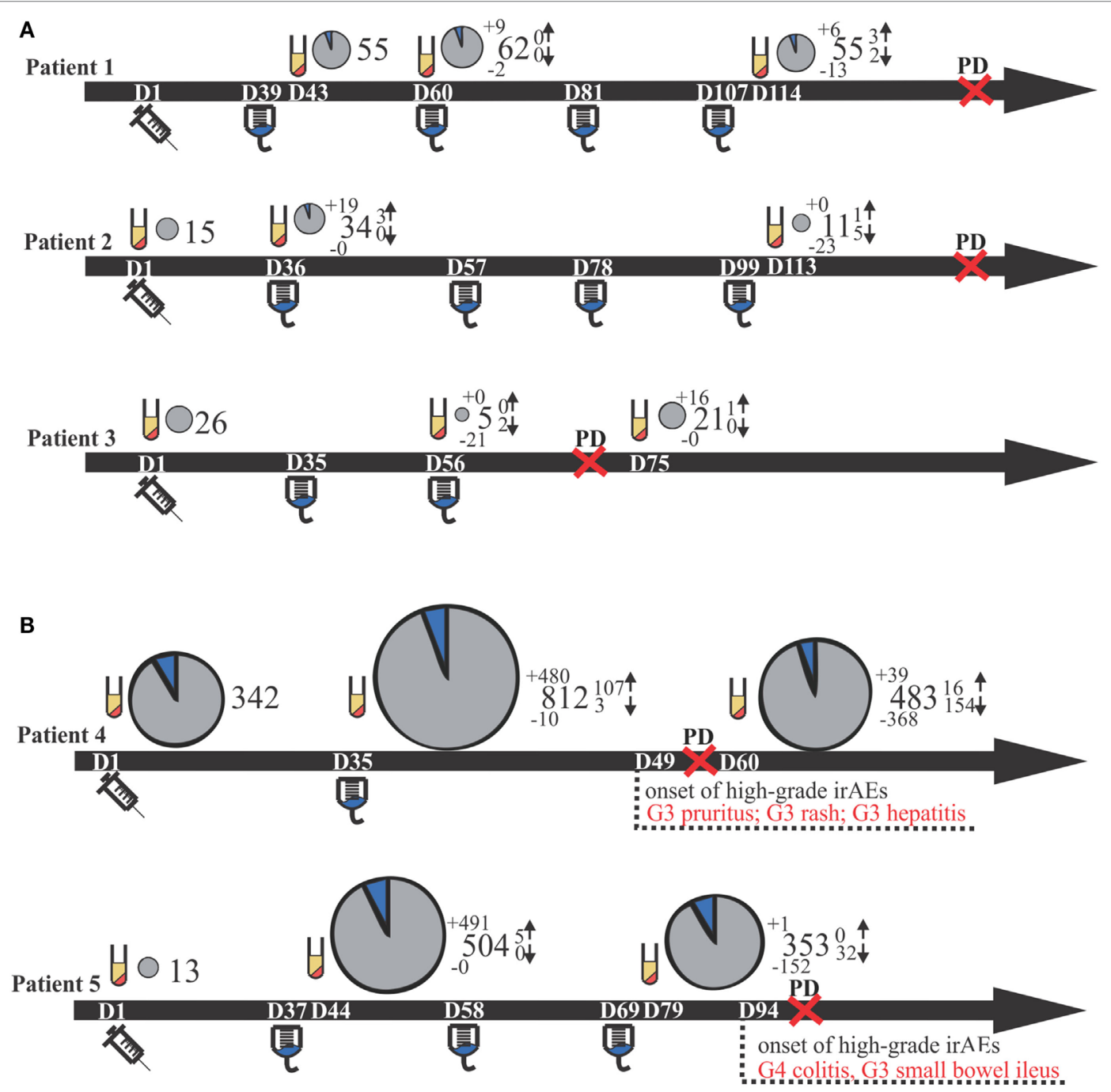

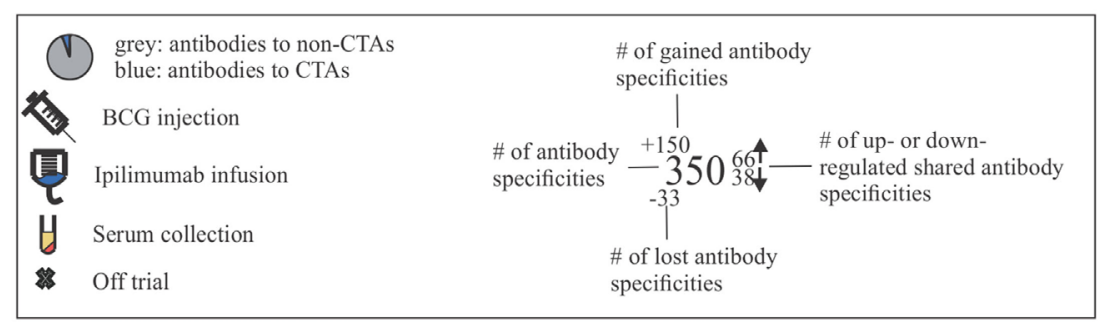

FIGURE 3 | Clinical time lines for all patients, including comparative size-proportional pie charts representing the number of antigens toward which antibody titers were detected. Time lines are separated by patients who did not develop high-grade irAEs (A) versus those who did (B). Dn, day n; PD, progressive disease; CTAs, cancer-testis antigens; Gn, grade n; irAEs, immune-related adverse events.

pretreatment existing enhanced reactivity. Autoantibody profiles did not overlap between patients, with unique antigen targets being detected in each patient. In addition, despite the high-grade irAEs being reported in two different organs, the liver and bowel, it is unclear whether there are apparent organotypic differences in the autoantibody repertoires of these patients due to limited patient numbers.

\section{DISCUSSION}

Clinical trials, which combine IL immune-stimulants with ICI, aim to extend the effectiveness of cancer immunotherapy by recruiting responses against additional cancer antigens within the injected tumor. This is the first such trial to evaluate BCG, a historically validated IL therapy, with ipilimumab in patients with 


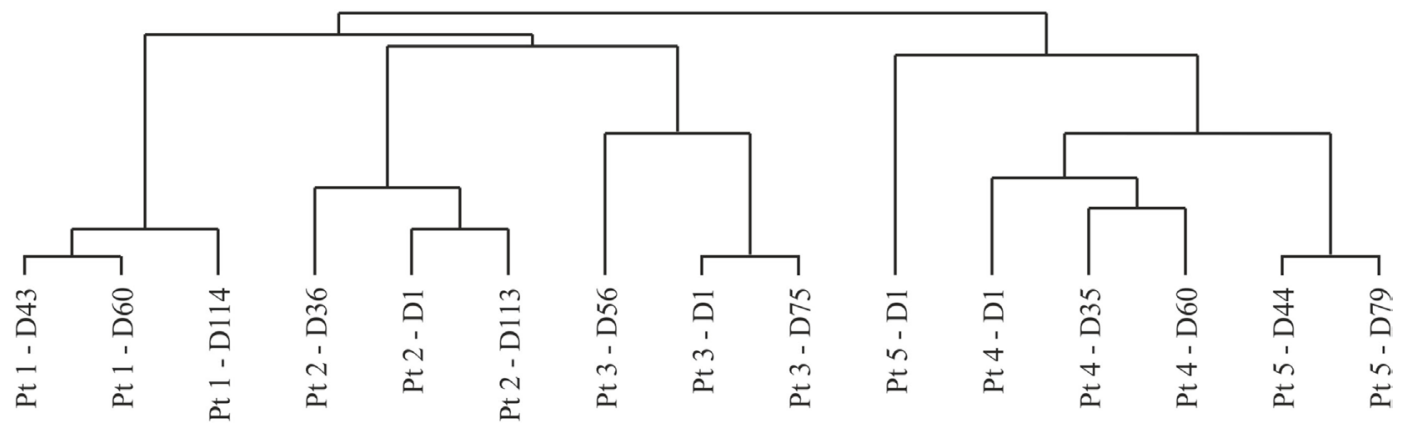

FIGURE 4 | Dendrogram resulting from hierarchical clustering using the Spearman rank correlation method with average linkage. Dn, day n; Pt, patient.

advanced melanoma. Although IL BCG was well tolerated, those patients treated with the higher dose of BCG $\left(0.80-3.20 \times 10^{6} \mathrm{cfu}\right)$ developed high-grade irAEs following ipilimumab. All irAEs were typical of ipilimumab and managed as per standardized treatment algorithms. We propose that BCG may have contributed to enhanced immune activation and therefore higher-grade irAEs. These irAEs together with the availability of anti-programmed death 1 (PD-1) therapies as first-line therapy at our institution resulted in slow patient enrollment and early termination of the trial; however, our preliminary correlative seromic analyses of patients with severe irAEs suggest the potential value of serology as a diagnostic tool to anticipate and evaluate autoimmune toxicity.

As the treatment of advanced melanoma continues to evolve, combination and/or sequential treatments with immunotherapy, targeted therapy, chemotherapy, radiotherapy, and even surgery are being trialed to harness an antitumor immune response to obtain maximum clinical benefit. Talimogene laherparepvec is the first oncolytic virus approved by the U.S. Food and Drug Administration for the local treatment of unresectable lesions in patients with recurrent melanoma. Approval was based on the phase III study, OPTiM, which demonstrated significant improvement in responses in injected and uninjected lesions versus subcutaneous GM-CSF (29). Recent studies combining this oncolytic virus with pembrolizumab have demonstrated high overall (62\%) and complete response (33\%) rates in advanced melanoma (30). Ipilimumab has been evaluated in combination with vaccination $(31,32)$. In one study $(31)$, ipilimumab in combination with a peptide vaccine resulted in two complete responses and five partial responses among 56 patients with progressive stage IV melanoma, with each of these responses shown to be durable. In another trial (32), patients with resected stage IIIC/ IV melanoma received ipilimumab plus a multipeptide vaccine; $25 \%$ of patients had grades 3-4 irAEs that were dose-limiting and 27 of 75 patients relapsed after a median follow-up of 23 months. While several such combinations of localized plus systemic immunotherapies offer promise, many unselected patients with a higher disease burden or more rapidly progressive disease still face poor outcomes with existing immunotherapy combinations.

The onset of irAEs resembling classic autoimmunity remains a limitation in cancer immunotherapy (33), and while generally manageable when immunotherapeutics are administered as single agents, the incidence can increase substantially when immunotherapeutics are combined (34). Developing strategies that rationally combine immunotherapeutic agents to minimize toxicities and maximize efficacy is therefore an important area of ongoing investigation. While the identification of predictive biomarkers for therapeutic efficacy is actively being pursued (35-39), comparatively little attention has been placed on identifying reliable biomarkers that can predict adverse autoimmune events.

In this study, we investigated the autoantibody repertoire of a small number of trial patients using the Immunome ${ }^{\mathrm{TM}}$ protein array that can identify serological responses to over 1,600 human proteins. We found that the two patients who developed clinically severe autoimmunity had an accompanying profound serological signature, reflecting immune reactivity against a broad panel of autoantigens. Indeed, patients experiencing high-grade irAEs could readily be distinguished on the basis of these autoantibodies which were reactive against almost half of the proteins on the array. Importantly, these elevated autoantibody specificity counts preceded the development of clinically evident autoimmunity. Despite the very limited patient numbers in this pilot trial, it is tempting to speculate that measuring the autoantibody repertoire of cancer patients at risk of experiencing irAEs may predict the development of toxicity.

The breadth of this autoimmune reactivity was suggestive of a systemic B cell deregulation, rather than a focused immune response, as might typically occur following an infection or vaccine. Similar responses have been reported in chronic humoral rejection of organ transplants (40). We postulate that the chronic inflammatory conditions associated with interstitial mycobacterial infection resulted in the indiscriminate release of both cancer antigens and autoantigens that were not cancer-specific. In the presence of an ICI, both anticancer immunity and autoimmunity were enhanced. This is in accordance with the proposed notion that the disruption of immune tolerance and the onset of inflammation can enhance autoantibody production (41). There was no sign of clinical activity, so the reactivity against cancer-specific targets did not appear to be clinically useful; however, with a small number of patients, it is difficult to be certain. This specific immunotherapy combination will not be pursued further due to the apparent excess of clinical toxicity. Nonetheless, larger studies-likely in the setting of more tolerable immunotherapy combinations-will be required to validate these seromic findings and to better understand the involved mechanisms. 
In conclusion, dose escalation of IL BCG followed by ipilimumab therapy in a pilot trial of limited patient numbers was not well tolerated in advanced melanoma patients and showed no evidence of clinical benefit. Whether the onset of the observed high-grade irAEs was enhanced by IL BCG or simply a result of ipilimumab alone remains unclear. Nonetheless, investigating humoral immunity may offer a means to detect the early onset of a wide spectrum of irAEs in cancer patients treated with immunotherapy, warranting further larger studies.

\section{ETHICS STATEMENT}

This study (ClinicalTrials.gov number NCT01838200) was conducted at Austin Health, Heidelberg, Australia, and was approved by the institution's human research ethics committee. Written informed consent was obtained from all patients, and all methods were performed in accordance with the protocol-specified guidelines and regulations.

\section{AUTHOR CONTRIBUTIONS}

$\mathrm{JD}, \mathrm{AB}$, and JC were responsible for the immunome array study design. JD was responsible for the data analysis, representation, and interpretation. JB, $\mathrm{AB}$, and JC provided additional expert input into the key findings. MA and JC were responsible for the planning and execution of the clinical trial. SP and MA provided relevant clinical information. KW, AP, CT, and SO

\section{REFERENCES}

1. Luke JJ, Flaherty KT, Ribas A, Long GV. Targeted agents and immunotherapies: optimizing outcomes in melanoma. Nat Rev Clin Oncol (2017) 14(8):463-82. doi:10.1038/nrclinonc.2017.43

2. Schadendorf D, Hodi FS, Robert C, Weber JS, Margolin K, Hamid O, et al. Pooled analysis of long-term survival data from phase II and phase III trials of ipilimumab in unresectable or metastatic melanoma. J Clin Oncol (2015) 33(17):1889-94. doi:10.1200/JCO.2014.56.2736

3. Sanderson K, Scotland R, Lee P, Liu D, Groshen S, Snively J, et al. Autoimmunity in a phase I trial of a fully human anti-cytotoxic T-lymphocyte antigen-4 monoclonal antibody with multiple melanoma peptides and montanide ISA 51 for patients with resected stages III and IV melanoma. J Clin Oncol (2005) 23(4):741-50. doi:10.1200/JCO.2005.01.128

4. Hodi FS, O'day SJ, McDermott DF, Weber RW, Sosman JA, Haanen JB, et al. Improved survival with ipilimumab in patients with metastatic melanoma. $N$ Engl J Med (2010) 2010(363):711-23. doi:10.1056/NEJMoa1003466

5. Robert C, Thomas L, Bondarenko I, O'day S, Weber J, Garbe C, et al. Ipilimumab plus dacarbazine for previously untreated metastatic melanoma. N Engl J Med (2011) 364(26):2517-26. doi:10.1056/NEJMoa1104621

6. Morton DL, Foshag LJ, Nizze JA, Gupta RK, Famatiga E, Hoon DS, et al. Active specific immunotherapy in malignant melanoma. Semin Surg Oncol (1989) 5(6):420-5. doi:10.1002/ssu.2980050608

7. Morton DL, Eilber FR, Holmes EC, Ramming KP. Preliminary results of a randomized trial of adjuvant immunotherapy in patients with malignant melanoma who have lymph node metastases. Aust N Z J Surg (1978) 48(1):49-52. doi:10.1111/j.1445-2197.1978.tb05804.x

8. Morton DL, Eilber FR, Holmes EC, Sparks FC, Ramming K. BCG immunotherapy as a systemic adjunct to surgery in malignant melanoma. Med Clin North Am (1976) 60(3):431-9. doi:10.1016/S0025-7125(16)31890-9

9. Morton DL, Eilber FR, Holmes EC, Hunt JS, Ketcham AS, Silverstein MJ, et al. BCG immunotherapy of malignant melanoma: summary of a seven-year experience. Ann Surg (1974) 180(4):635-43. doi:10.1097/00000658-19741000000029 were responsible for clinical sample processing and additional immunology experiments that were excluded from this article. JD wrote the manuscript, and SP, MA, JB, AB, and JC reviewed and edited. All authors read and approved the submitted version.

\section{ACKNOWLEDGMENTS}

We thank the Ludwig Institute for Cancer Research (study sponsor) and Bristol-Myers Squibb for funding this clinical study, as well as all patients who volunteered to participate. We also thank Rodica Stan, Tina Cavicchiolo, and Noel Micallef for their essential contributions to the clinical trial. JD is supported by a Tour de Cure Scott Canner Young Research Grant. MA is supported by a NHMRC CJ Martin Fellowship (\#1148680). JB is supported by a Research Chair Grant from the South African National Research Foundation (\#64760). AB is supported by a Mid-Career Research Fellowship from the Victorian Cancer Agency (\#17019). The Olivia Newton-John Cancer Research Institute acknowledges the support of the Victorian Government Operational Infrastructure Support Program.

\section{SUPPLEMENTARY MATERIAL}

The Supplementary Material for this article can be found online at http://www.frontiersin.org/articles/10.3389/fimmu.2018.00411/ full\#supplementary-material.

10. Pinsky CM, Hirshaut Y, Oettgen HF. Treatment of malignant melanoma by intratumoral injection of BCG. Natl Cancer Inst Monogr (1973) 39:225-8.

11. Brosman SA. The use of bacillus Calmette-Guérin in the therapy of bladder carcinoma in situ. J Urol (1985) 134(1):36-9. doi:10.1016/S0022-5347(17)46969-2

12. Herr HW, Pinsky CM, Whitmore WF Jr, Oettgen HF, Melamed MR. Effect of intravesical bacillus Calmette-Guérin (BCG) on carcinoma in situ of the bladder. Cancer (1983) 51(7):1323-6. doi:10.1002/10970142(19830401)51:7<1323::AID-CNCR2820510724>3.0.CO;2-2

13. Kelley DR, Ratliff TL, Catalona WJ, Shapiro A, Lage JM, Bauer WC, et al. Intravesical bacillus Calmette-Guérin therapy for superficial bladder cancer: effect of bacillus Calmette-Guérin viability on treatment results. J Urol (1985) 134(1):48-53. doi:10.1016/S0022-5347(17)46976-X

14. Hsueh EC, Famatiga E, Shu S, Ye X, Morton DL. Peripheral blood CD4 ${ }^{+}$T-cell response before postoperative active immunotherapy correlates with clinical outcome in metastatic melanoma. Ann Surg Oncol (2004) 11(10):892-9. doi:10.1245/ASO.2004.02.018

15. Morton D, Eilber FR, Malmgren RA, Wood WC. Immunological factors which influence response to immunotherapy in malignant melanoma. Surgery (1970) 68(1):158-63; discussion 63-4.

16. Nathanson L, Schoenfeld D, Regelson W, Colsky J, Mittelman A. Prospective comparison of intralesional and multipuncture BCG in recurrent intradermal melanoma. Cancer (1979) 43(5):1630-5. doi:10.1002/10970142(197905)43:5<1630::AID-CNCR2820430511>3.0.CO;2-Z

17. Sparks FC, Silverstein MJ, Hunt JS, Haskell CM, Pilch YH, Morton DL. Complications of BCG immunotherapy in patients with cancer. $N$ Engl J Med (1973) 289(16):827-30. doi:10.1056/NEJM197310182891603

18. Hsueh EC, Essner R, Foshag LJ, Ye W, Morton DL. Active immunotherapy by reinduction with a polyvalent allogeneic cell vaccine correlates with improved survival in recurrent metastatic melanoma. Ann Surg Oncol (2002) 9(5):486-92. doi:10.1007/BF02557273

19. Hsueh EC, Gupta RK, Qi K, Morton DL. Correlation of specific immune responses with survival in melanoma patients with distant metastases receiving polyvalent melanoma cell vaccine. J Clin Oncol (1998) 16(9):2913-20. doi:10.1200/JCO.1998.16.9.2913 
20. Vilella R, Benitez D, Mila J, Vilalta A, Rull R, Cuellar F, et al. Treatment of patients with progressive unresectable metastatic melanoma with a heterologous polyvalent melanoma whole cell vaccine. Int J Cancer (2003) 106(4):626-31. doi:10.1002/ijc.11242

21. Morton DL, Mozzillo N, Thompson JF, Kelley MC, Faries M, Wagner J, et al. An international, randomized, phase III trial of bacillus Calmette-Guérin (BCG) plus allogeneic melanoma vaccine (MCV) or placebo after complete resection of melanoma metastatic to regional or distant sites. J Clin Oncol (2007) 25(Suppl 18):8508. doi:10.1200/jco.2007.25.18_suppl.8508

22. Beatty GL, Gladney WL. Immune escape mechanisms as a guide for cancer immunotherapy. Clin Cancer Res (2015) 21(4):687-92. doi:10.1158/10780432.CCR-14-1860

23. Duarte J, Blackburn J. Advances in the development of human protein microarrays. Expert Rev Proteomics (2017) 14(7):627-41. doi:10.1080/14789 450.2017.1347042

24. Eisenhauer EA, Therasse P, Bogaerts J, Schwartz LH, Sargent D, Ford R, et al. New response evaluation criteria in solid tumours: revised RECIST guideline (version 1.1).Eur J Cancer (2009) 45(2):228-47. doi:10.1016/j.ejca.2008.10.026

25. Wolchok J, Hoos A, O’Day S, Weber J, Hamid O, Lebbé C, et al. Guidelines for the evaluation of immune therapy activity in solid tumors: immune-related response criteria. Clin Cancer Res (2009) 15(23):7412-20. doi:10.1158/10780432.CCR-09-1624

26. Suwarnalata G, Tan A, Isa H, Gudimella R, Anwar A, Loke M, et al. Augmentation of autoantibodies by Helicobacter pylori in Parkinson's disease patients may be linked to greater severity. PLoS One (2016) 11(4):e0153725. doi:10.1371/journal.pone.0153725

27. Duarte J, Serufuri J-M, Mulder N, Blackburn J. Protein function microarrays: design, use and bioinformatic analysis in cancer biomarker discovery and quantitation. In: Wang X, editor. Bioinformatics of Human Proteomics. Dordrecht: Springer (2013). p. 39-74.

28. Simpson AJG, Caballero O, Jungbluth A, Chen Y-T, Old L. Cancer/testis antigens, gametogenesis and cancer. Nat Rev Cancer (2005) 5(8):615-25. doi:10.1038/nrc1669

29. Andtbacka RH, Kaufman HL, Collichio F, Amatruda T, Senzer N, Chesney J, et al. Talimogene laherparepvec improves durable response rate in patients with advanced melanoma. J Clin Oncol (2015) 33(25):2780-8. doi:10.1200/JCO.2014.58.3377

30. Ribas A, Dummer R, Puzanov I, VanderWalde A, Andtbacka RHI, Michielin $\mathrm{O}$, et al. Oncolytic virotherapy promotes intratumoral T cell infiltration and improves anti-PD-1 immunotherapy. Cell (2017) 170(6):1109.e-19.e. doi:10.1016/j.cell.2017.08.027

31. Attia P, Phan GQ, Maker AV, Robinson MR, Quezado MM, Yang JC, et al. Autoimmunity correlates with tumor regression in patients with metastatic melanoma treated with anti-cytotoxic T-lymphocyte antigen-4. J Clin Oncol (2005) 23(25):6043-53. doi:10.1200/JCO.2005.06.205
32. Weber J, Targan S, Scotland R, Snively J, Garcia M, Yellin M, et al. Phase II trial of extended dose anti-CTLA-4 antibody ipilimumab (formerly MDX-010) with a multi-peptide vaccine for resected stages IIIC and IV melanoma. J Clin Oncol (2006) 24(18_suppl):2510. doi:10.1200/jco.2006.24.18_suppl.2510

33. June $\mathrm{CH}$, Warshauer JT, Bluestone JA. Is autoimmunity the Achilles' heel of cancer immunotherapy? Nat Med (2017) 23(5):540-7. doi:10.1038/ nm. 4321

34. Larkin J, Chiarion-Sileni V, Gonzalez R, Grob JJ, Cowey CL, Lao CD, et al. Combined nivolumab and ipilimumab or monotherapy in untreated melanoma. N Engl J Med (2015) 373(1):23-34. doi:10.1056/NEJMoa1504030

35. Ayers M, Lunceford J, Nebozhyn M, Murphy E, Loboda A, Kaufman DR, et al. IFN-gamma-related mRNA profile predicts clinical response to $\mathrm{PD}-1$ blockade. J Clin Invest (2017) 127(8):2930-40. doi:10.1172/JCI91190

36. Gibney G, Weiner L, Atkins M. Predictive biomarkers for checkpoint inhibitor-based immunotherapy. Lancet Oncol (2016) 17(12):e542-51. doi:10.1016/ S1470-2045(16)30406-5

37. Hugo W, Zaretsky JM, Sun L, Song C, Moreno BH, Hu-Lieskovan S, et al. Genomic and transcriptomic features of response to anti-PD-1 therapy in metastatic melanoma. Cell (2016) 165(1):35-44. doi:10.1016/j.cell.2016.02.065

38. Patel S, Kurzrock R. PD-L1 expression as a predictive biomarker in cancer immunotherapy. Mol Cancer Ther (2015) 14(4):847-56. doi:10.1158/15357163.MCT-14-0983

39. Topalian S, Taube J, Anders R, Pardoll D. Mechanism-driven biomarkers to guide immune checkpoint blockade in cancer therapy. Nat Rev Cancer (2016) 16(5):275-87. doi:10.1038/nrc.2016.36

40. Porcheray F, DeVito J, Yeap B, Xue L, Dargon I, Paine R, et al. Chronic humoral rejection of human kidney allografts associates with broad autoantibody responses. Transplantation (2010) 89(10):1239-46. doi:10.1097/ TP.0b013e3181d72091

41. Zaenker P, Gray ES, Ziman MR. Autoantibody production in cancer-the humoral immune response toward autologous antigens in cancer patients. Autoimmun Rev (2016) 15(5):477-83. doi:10.1016/j.autrev.2016.01.017

Conflict of Interest Statement: JB is a consultant for Sengenics and developed their Immunome ${ }^{\mathrm{TM}}$ protein array product while being an academic researcher at the University of Cambridge. No additional authors have a conflict of interest to declare.

Copyright (c) 2018 Da Gama Duarte, Parakh, Andrews, Woods, Pasam, Tutuka, Ostrouska, Blackburn, Behren and Cebon. This is an open-access article distributed under the terms of the Creative Commons Attribution License (CC BY). The use, distribution or reproduction in other forums is permitted, provided the original author(s) and the copyright owner are credited and that the original publication in this journal is cited, in accordance with accepted academic practice. No use, distribution or reproduction is permitted which does not comply with these terms. 